\title{
Biomarker bei ambulant erworbener Pneumonie - was haben wir aus CAPNETZ gelernt? Ergebnisse aus dem Deutschen Kompetenznetz CAPNETZ
}

\author{
Biomarkers in Community Acquired Pneumonia - What did we Learn from the CAPNETZ Study? \\ Results from the German Competence Network CAPNETZ
}

Autoren

Institute

\author{
S. Krüger ${ }^{1}$, M. W. Pletz ${ }^{2}$, G. Rohde ${ }^{3}$
}

Medizinische Klinik I, Universitätsklinikum RWTH Aachen

Medizinische Klinik II, Universitätsklinikum Jena

Department of Respiratory Medicine, MUMC+, Maastricht eingereicht 13.10.2010

akzeptiert 18.10.2010

Bibliografie

Dol http://dx.doi.org/

10.1055/s-0030-1255935

Online-Publikation: 29.11.2010

Pneumologie 2011; 65:

110-113 @ Georg Thieme

Verlag KG Stuttgart · New York

ISSN 0934-8387

Korrespondenzadresse

Priv.-Doz. Dr. med.

Stefan Krüger

Medizinische Klinik I

Universitätsklinikum RWTH

Aachen

Pauwelsstr. 30

52057 Aachen

stkrueger@ukaachen.de

\section{Zusammenfassung \\ V}

Hintergrund: Bei der ambulant erworbenen Pneumonie (community acquired pneumonia $=$ CAP) wurden in den letzten Jahren Biomarker intensiv untersucht. Im Rahmen der CAPNETZStudie hatten wir die einmalige Gelegenheit, multizentrisch an einer großen Patientenzahl den Wert alter und neuer Biomarker für die CAP zu untersuchen.

Ergebnisse: In mehreren Substudien konnten wir Folgendes herausfinden: Die Werte für Procalcitonin, CRP und Leukozyten sind am höchsten bei typischer bakterieller Ätiologie, erlauben jedoch keinen Rückschluss auf die individuelle Ätiologie eines einzelnen Patienten. Die Serumspiegel der Biomarker sind oftmals höher bei Patienten ohne antibiotische Vortherapie, verglichen mit Patienten mit antibiotischer Vortherapie. Kardiovaskuläre neuere Biomarker sind gute Prädiktoren für die Kurz- und Langzeitmortalität der CAP, dabei den rein inflammatorischen Markern Procalcitonin, CRP und Leukozyten überlegen und dem klinischen CRB-65-Score mindestens vergleichbar. Pro-Adrenomedullin ist unter den neuen Biomarkern derjenige mit der besten prognostischen Wertigkeit.

Schlussfolgerungen: Biomarker korrelieren mit dem Schweregrad der CAP, erlauben aber keine individuelle Prädiktion der Ätiologie. Neuere kardiovaskuläre Biomarker sind geeignet für die Prognosebeurteilung der Kurz- und Langzeitmortalität der CAP. Die Kombination mehrerer Biomarker, die jeweils andere pathophysiologische Signalwege abbilden, hat das Potenzial, zukünftig das Management der CAP zu verbessern.

\section{Abstract}

$\nabla$

Background: Biomarkers have been intensively studied in community-acquired pneumonia (CAP) in recent years. In the context of the CAPNETZ study we had the unique opportunity to evaluate old and new biomarkers in a multicentre study with a high number of patients.

Results: In several substudies we found the following results: procalcitonin, CRP and leukocytes show highest values in patients with typical bacterial etiology of CAP, but do not allow individual prediction of etiology. Patients without antibiotic pre-treatment show higher values of biomarkers compared to patients with antibiotic pre-treatment. New cardiovascular biomarkers are good predictors for short- and long-term mortality in CAP, superior to the inflammatory markers procalcitonin, CRP and leukocytes and at least comparable to the clinical CRB-65 score. Pro-Adrenomedullin is among the new biomarkers the one with the best prognostic value.

Conclusions: Biomarkers correlate with the severity of CAP but do not allow individual prediction of etiology. New cardiovascular biomarkers are suitable for the evaluation of short- and longterm prognosis in CAP. The combination of several biomarkers reflecting different pathophysiological pathways has the potential to improve management of CAP in the future. 


\section{Einleitung}

Klinisch-chemische Parameter sind in den letzten Jahrzehnten Standard in der Diagnostik geworden, wie zum Beispiel Elektrolyte, Retentionswerte oder Transaminasen. Im Gegensatz zu diesen relativ unspezifischen Parametern, die sich bei einer Vielzahl von Erkrankungen verändern, haben sich in den letzten Jahren bei verschiedenen Krankheiten spezifischere Biomarker etabliert, die für Diagnose, Therapiesteuerung und Beurteilung der Prognose eingesetzt werden können. Das beste Beispiel hierfür ist das Troponin beim akuten Koronarsyndrom. Auch für pulmonale Infektionen inklusive der ambulant erworbenen Pneumonie (community-acquired pneumonia = CAP) wurden in den letzten Jahren Biomarker intensiv untersucht. Im Rahmen der CAPNETZStudie als groß angelegte Observationsstudie hatten wir die einmalige Gelegenheit, multizentrisch an einer großen Patientenzahl den Wert alter und neuer Biomarker für die Diagnostik und Prognoseabschätzung der CAP zu untersuchen.

\section{Biomarker und Ätiologie der CAP \\ $\nabla$}

Studie: Die inflammatorischen Marker C-reaktives Protein (CRP) und die Leukozytenzahl werden weit verbreitet in der Diagnostik von pulmonalen Infektionen verwendet. Ihre Nachteile sind jedoch, dass sie unspezifisch sind und die Differenzierung einer bakteriellen, atypischen oder viralen Ätiologie der CAP nicht erlauben. Procalcitonin (PCT) scheint hierfür eine vielversprechende Alternative zu sein. Das Serum PCT steigt bei bakteriellen Infektionen rasch an, während es bei viralen Erkrankungen und autoimmunen Syndromen niedrig bleibt. Ziel der Studie war es, die Korrelation der inflammatorischen Marker PCT, CRP und Leukozyten in Bezug auf die mikrobiologische Ätiologie zu evaluieren [1].

Wir schlossen 1337 Patienten mit CAP ein. Immunsupprimierte Patienten, definiert als Zustand nach Organ- oder Knochenmarktransplantation, zytostatischer oder immunsuppressiver Therapie, HIV-Infektion und/oder bekannte Immundefizienzsyndrome, wurden in CAPNETZ nicht eingeschlossen.

Eine intensive mikrobiologische Diagnostik, bestehend aus Blutkultur, Rachenspülwasser für den Nachweis von Viren mittels PCR, Sputumkultur, Urin für Legionellen- und PneumokokkenAntigen-Test sowie Antikörper-Bestimmungen, wurde durchgeführt. Bei allen Patienten wurden PCT, CRP, Leukozyten und der klinische CRB-65-Score bestimmt. Die Patienten wurden entsprechend der mikrobiologischen Ätiologie und des CRB-65Scores klassifiziert. Bei Patienten mit typischen bakteriellen Erregern waren die Spiegel von PCT, CRP und Leukozyten signifikant höher, verglichen mit einer atypischen (Mykoplasmen, Legionellen, Chlamydien) oder einer viralen Ätiologie. Ein PCT-Grenzwert von $0,1 \mathrm{ng} / \mathrm{mL}$ zeigte eine Odds Ratio von 8,3 und ein PCT-Grenzwert von $0,25 \mathrm{ng} / \mathrm{mL}$ eine Odds Ratio von 3,2, um eine S. pneumoniae CAP von anderen Formen der CAP mit atypischer oder viraler Ätiologie zu differenzieren. Es gab keine signifikanten Unterschiede bei PCT, CRP und Leukozyten bei Patienten mit atypischer oder viraler Ätiologie der CAP. Im Gegensatz zu CRP und Leukozyten stieg das PCT deutlich mit zunehmender Schwere der CAP an. CRB-65-Score, PCT, CRP und Leukozyten waren höher bei hospitalisierten Patienten im Vergleich zu ambulant behandelten Patienten, wobei aber nur der CRB-65-Score und das PCT eine gute Diskriminationsfähigkeit zeigten hinsichtlich der Hospitalisation.
Schlussfolgerungen: PCT, CRP und Leukozyten sind am höchsten bei einer typischen bakteriellen Ätiologie der CAP, erlauben jedoch keine individuelle Prädiktion der Ätiologie.

Fazit für die Praxis: Leider ist es aufgrund der großen Überlappung der Messwerte der einzelnen Biomarker bei typischer, atypischer und viraler Ätiologie nicht möglich, für den einzelnen Patienten eine Ätiologie der CAP auf der Basis einer BiomarkerMessung vorherzusagen. Deshalb können die inflammatorischen Biomarker auch nicht für die Wahl des individuell richtigen Antibiotikums herangezogen werden. Eine wesentliche Ursache für dieses Dilemma liegt darin, dass die Höhe der Biomarker nicht nur von der Ätiologie, sondern insbesondere auch von der Schwere der CAP abhängt. So sind hohe PCT-Werte z.B. indikativ für eine S. pneumoniae-Ätiologie auf der einen Seite, aber auch für eine schwere CAP (evtl. ohne mikrobiologischen Erregernachweis) auf der anderen Seite. Deshalb bleibt die Verwendung etablierter und validierter klinischer Scores, wie z. B. des CRB-65Scores, eine praktische Notwendigkeit zur Erkennung der Schwere der CAP und der daraus abzuleitenden Therapiemaßnahmen [2].

\section{Biomarker und antibiotische Therapie $\nabla$}

Unter einer effektiven Antibiotika-Therapie der CAP fallen die Werte der inflammatorischen Parameter ab, sodass deren Verlauf zur Therapiekontrolle empfohlen wird. In der Notaufnahme der Kliniken stellen sich jedoch viele Patienten vor, die schon zuvor ambulant antibiotisch behandelt worden sind. Aus dem CAPNETZ-Kollektiv wissen wir, dass dies ca. 25\% der Patienten sind, die in der Klinik behandelt werden. Über den Einfluss der antibiotischen Vortherapie auf den klinischen Schweregrad und die Interpretation der inflammatorischen Biomarker gab es bislang keine Daten. Unser Ziel war deshalb, den Einfluss der antibiotischen Vortherapie auf die Serumspiegel der inflammatorischen Marker PCT, CRP und Leukozyten sowie kardiovaskulären Biomarker pro-Arginin-Vasopressin (Copeptin) und pro-Atrial-Natriuretisches Peptid (proANP) bei Krankenhausaufnahme zu evaluieren [3-5].

Studie 1: In einer ersten Studie wurden 370 hospitalisierte Patienten mit CAP eingeschlossen [3]. Bei Aufnahme wurden die Spiegel des kardiovaskulären Biomarkers Copeptin und der inflammatorischen Marker PCT, CRP und Leukozyten sowie der klinisch etablierte CRB-65-Score bestimmt. 85 Patienten hatten eine antibiotische Vortherapie und 285 Patienten hatten keine. Die Copeptin-Spiegel stiegen mit zunehmender Schwere der CAP bei Patienten ohne antibiotische Vortherapie an, aber nicht bei Patienten mit antibiotischer Vortherapie. Patienten mit antibiotischer Vortherapie zeigten signifikant niedrigere Spiegel von Copeptin und PCT, aber nicht von CRP und Leukozyten, verglichen mit den Patienten ohne antibiotische Vortherapie.

Studie 2: In eine zweite Studie wurden 991 hospitalisierte Patienten mit CAP eingeschlossen [4]. Bei Aufnahme wurden die Spiegel der inflammatorischen Marker PCT, CRP und Leukozyten sowie der klinisch etablierte CRB-65-Score bestimmt. 232 Patienten hatten eine antibiotische Vortherapie und 759 Patienten hatten keine. Patienten mit antibiotischer Vortherapie zeigten signifikant niedrigere Spiegel von PCT und Leukozyten, aber nicht von CRP, verglichen mit den Patienten ohne antibiotische Vortherapie. 
Schlussfolgerungen: Die Serumspiegel von Copeptin und PCT, aber nicht von CRP, sind höher bei Patienten ohne antibiotische Vortherapie, verglichen mit Patienten mit antibiotischer Vortherapie. Copeptin und PCT sind bei CAP somit sensitivere Biomarker mit dynamischerer Kinetik, die entsprechend rascher auf eine Therapie reagieren als die üblichen Marker CRP und Leukozyten. Fazit für die Praxis: Eine antibiotische Vortherapie sollte bei der korrekten Interpretation von kardiovaskulären (Copeptin, atrial natriuretisches Peptid) und inflammatorischen Biomarkern (PCT) berücksichtigt werden. Dies ist wichtig, um die Kinetik der Biomarker unter antibiotischer Therapie richtig einzuschätzen und daraus die adäquaten diagnostischen und therapeutischen Schlüsse zu ziehen.

\section{Biomarker und Prognose \\ $\nabla$}

Die etablierten Risiko-Scores für die CAP sind der komplizierte PSI- und der einfache CRB-65-Score. Die Scores sind aber nur für die Abschätzung des Risikos einer Kurzzeitmortalität validiert. Bei Patienten, die eine CAP überlebt haben, zeigt sich jedoch auch im langfristigen Verlauf eine erhöhte Sterblichkeit, die jene der altersentsprechenden Bevölkerung deutlich übersteigt. Bislang gibt es keinen Score und auch keinen etablierten Biomarker für die Abschätzung der Langzeitprognose nach CAP.

Studien 1 und 2 (Kurzzeitmortalität): In der ersten Studie haben wir uns damit beschäftigt, ob die inflammatorischen Marker Leukozyten, CRP und PCT einen prognostischen Wert haben für die Abschätzung der Kurzzeitmortalität nach 28 Tagen [6,7]. Dabei konnten wir an einem Kollektiv von 1671 Patienten herausfinden, dass der prognostische Wert des PCT vergleichbar mit dem CRB-65-Score und besser als die etablierten inflammatorischen Marker CRP und Leukozyten ist [6]. In einer zweiten Studie mit Analyse der neuen kardiovaskulären Marker Copeptin und proANP konnten wir an 589 Patienten zeigen, dass proANP und Copeptin für die Beurteilung der Kurzzeitprognose bei CAP dem CRB-65-Score mindestens vergleichbar sind [7].

Studie 3 (Langzeitmortalität): Die interessanten Ergebnisse dieser beiden ersten Studien bezüglich der Kurzzeitmortalität waren Anlass, in einer Folgestudie an einem größeren Kollektiv die Wertigkeit der Biomarker für die Prädiktion der Kurzzeit- und der Langzeitmortalität nach 180 Tagen zu untersuchen [8]. Wir schlossen 1740 Patienten ein. Die Biomarker proANP, Copeptin, PCT, CRP, Leukozyten und der CRB-65-Score wurden bei Aufnahme bestimmt. Die Patienten wurden über 180 Tage nachbeobachtet. ProANP- und Copeptin-Spiegel stiegen mit zunehmendem Schweregrad der CAP an. 78 Patienten (4,5\%) starben innerhalb von 28 Tagen und zusätzlich 93 Patienten (5,6\%) starben zwischen Tag 29 und 180. Bei Patienten, die innerhalb von 28 und 180 Tagen starben, lag der Median der Werte von proANP und Copeptin signifikant höher, verglichen mit den Werten der Überlebenden. In der ROC-Analyse für das 28- und 180-TageÜberleben waren die Area under the curve (AUC) für Copeptin $(0,84$ und 0,78$)$ und proANP $(0,81$ und 0,81$)$ höher, verglichen mit der AUC des CRB-65-Scores (0,74 und 0,71), PCT, CRP und Leukozyten. Der optimale Grenzwert zur Prädiktion des Todes nach 180 Tagen lag bei 102 pmol/L für proANP mit einer Sensitivität von $88 \%$, Spezifität von $62 \%$, positiv prädiktivem Wert von $20 \%$ und negativ prädiktivem Wert von $98 \%$ (Odds Ratio 11,9). Der optimale Grenzwert für Copeptin zur Prädiktion des Todes nach 180 Tagen war 22,3 pmol/L mit einer Sensitivität von 65\%, Spezifität von $80 \%$, positiv prädiktivem Wert von $26 \%$ und einem negativ prädiktiven Wert von 95\% (Odds Ratio 7,3). In der multivariablen Cox-Regressions-Analyse mit Adjustierung für Komorbidität und Schweregrad der Pneumonie waren das proANP und Copeptin unabhängige und die stärksten Prädiktoren der Kurzund Langzeitmortalität.

Schlussfolgerungen: Die kardiovaskulären Biomarker proANP und Copeptin sind neue, vielversprechende Parameter für die Prädiktion der Kurz- und der Langzeitmortalität von CAP-Patienten und dabei den inflammatorischen Markern und dem CRB-65 Score überlegen. Insbesondere der hohe negative prädiktive Wert bezüglich der Mortalität könnte für das zukünftige Management eine Rolle spielen.

Studie 4 (Langzeitmortalität): In der Zwischenzeit konnten andere Gruppen zeigen, dass weitere neuere Biomarker wie das proEndothelin-1 (proET-1) und das pro-Adrenomedullin (ADM) eine Rolle für die Prognosebeurteilung bei tiefen Atemwegsinfektionen spielen. Es war jedoch unklar, welcher der neueren Biomarker der beste ist zur Prognosebeurteilung bei der CAP, da es bislang keine Vergleichsstudien gab. Ziel unserer Studie war die Bestimmung aller neueren Biomarker (proANP, Copeptin, proADM, proET-1, PCT) zusammen mit den etablierten Markern CRP und Leukozyten und des CRB-65-Scores für die Prädiktion der Kurzzeit- (28 Tage) und Langzeitmortalität (180 Tage) bei allen Patienten sowie ein intraindividueller Vergleich [9]. Es wurden 785 Patienten eingeschlossen und über 180 Tage nachverfolgt. Die Spiegel von proADM, proANP, Copeptin, proET-1, PCT, CRP und Leukozyten stiegen mit zunehmender Schwere der Erkrankung entsprechend dem CRB-65-Score an. Bei Patienten, die innerhalb von 28 und 180 Tagen starben (2,5\% bzw. 5,1\%), waren proADM, proANP, proAVP, proET-1 und PCT signifikant höher, verglichen mit Überlebenden. In der Cox-Regressions-Analyse zeigte proADM die beste Performance für die Prädiktion des 28Tage- und 180-Tage-Überlebens. In der ROC-Analyse für die 28Tage-Mortalität war die AUC für proADM $(0,85)$ höher als bei proANP $(0,81)$, Copeptin $(0,80)$, proET- $1(0,79)$ und CRB-65-Score $(0,73)$. Für die Prädiktion der Langzeitmortalität nach 180 Tagen war die AUC von proADM $(0,79)$ signifikant höher als für proANP $(0,73)$, Copeptin $(0,72)$, proET-1 $(0,76)$, PCT, CRP und Leukozyten. ProADM war unabhängig vom CRB-65-Score und hatte einen zusätzlichen prognostischen Wert für die Prädiktion sowohl der Kurz- als auch der Langzeitmortalität.

Schlussfolgerungen: Alle neueren Biomarker waren gute Prädiktoren für die Kurz- und Langzeitmortalität der CAP, den rein inflammatorischen Markern PCT, CRP und Leukozyten überlegen und dem CRB-65-Score mindestens vergleichbar. ProADM war unter den neuen Biomarkern derjenige mit der besten prognostischen Wertigkeit und konnte in der Kombination mit dem CRB65-Score die Prädiktion der Kurz- und Langzeitmortalität signifikant verbessern. Ähnlich wie in der ersten Studie war die Langzeitmortalität nach 180 Tagen doppelt so hoch wie die Kurzzeitmortalität nach 28 Tagen.

Wie lässt sich erklären, dass kardiovaskuläre Biomarker bessere prognostische Aussagen liefern als inflammatorische Biomarker? Die neuen Biomarker spiegeln verschiedene Aspekte der CAP wider. Die inflammatorischen Marker PCT, CRP und Leukozyten dienen vorrangig der Diagnose der Infektion. Die kardiovaskulären Marker proANP und Copeptin sind bei Herzinsuffizienz und Sepsis erhöht. Endothelin ist ein sehr starker Vasokonstriktor und erhöht bei pulmonalen Infektionen, Hypoxie und pulmonaler Hypertonie. Adrenomedullin besitzt multiple Effekte wie Vasodilatation, Immunmodulation und bakterizide Aktivität. Die Haupttodesursachen nach einer CAP sind kardiovaskuläre Er- 
krankungen und Malignome, aber auch COPD, Niereninsuffizienz und Infektionen spielen eine wichtige Rolle. Eine Erklärung für die Übersterblichkeit der Patienten mit CAP könnte eine subklinische persistierende Inflammationsreaktion sein, die mit einer erhöhten kardiovaskulären Mortalität in der Folge assoziiert ist. Andererseits könnte die CAP in Zusammenhang mit einer zugrunde liegenden, aber noch nicht bekannten kardiovaskulären Erkrankung, Malignom oder Niereninsuffizienz auftreten. Ein häufiges Beispiel ist das Lungenkarzinom, das sich als poststenotische Pneumonie erstmalig manifestieren kann oder eine Stauungspneumonie bei Linksherzinsuffizienz. Wenn sich die Diagnostik bei der CAP wie bisher im Wesentlichen auf eine Röntgen-Thorax-Aufnahme, ein Basis-Labor mit Differenzialblutbild und CRP sowie ein EKG beschränkt, wird man viele kardiovaskuläre Begleiterkrankungen auch bei guter Anamnese nicht erkennen. Hier können die neuen Biomarker proANP, Copeptin, proET-1 und proADM einen wertvollen Beitrag leisten.

Fazit für die Praxis: Die Langzeitmortalität nach einer CAP ist exzessiv erhöht, was bisher insgesamt wenig Beachtung gefunden hat. Die CAP scheint ein Indikator für lebenslimitierende Grunderkrankungen zu sein. Nach diesen sollte bei Auftreten einer CAP intensiver gesucht werden. Aufgrund oftmals knapper Ressourcen, z.B. der Echokardiografie, ermöglicht die Bestimmung kardiovaskulärer Biomarker ein Screening und Identifikation von Hochrisikopatienten. Bei diesen sollte eine gezielte intensivere Diagnostik und ggf. eine Therapie der Grunderkrankung erfolgen. Die Biomarker ersetzen somit in keiner Weise den Arzt, können ihm aber helfen, zeitliche und apparative Ressourcen unter ökonomisch immer schwieriger werdenden Bedingungen besser zu nutzen und die Langzeitprognose der Patienten mit CAP zu verbessern.

\section{Zusammenfassung}

Durch CAPNETZ war es möglich, an sehr großen Patientenzahlen Biomarker-Studien durchzuführen. Hierdurch konnten wir erkennen, dass Biomarker uns leider nicht helfen können, die mikrobiologische Ätiologie einer CAP individuell sicher vorherzusagen. Auch werden die Biomarker durch eine antibiotische Vortherapie beeinflusst. Zur Diagnostik der Infektion werden inflammatorische Biomarker eingesetzt. Die im ambulanten Sektor und der Klinik sehr verbreiteten Marker CRP und Leukozyten korrelieren jedoch nicht sehr gut mit dem Schweregrad der Pneumonie und auch nicht mit der Prognose. Hierfür ist der für bakterielle Infektionen sehr viel spezifischere Biomarker PCT besser geeignet, wird aber bislang noch nicht flächendeckend eingesetzt. Dabei gibt es mittlerweile viele methodisch exzellente Studien, die zeigen konnten, dass durch einen PCT-gesteuerten Algorithmus die Dauer der Antibiotika-Therapie bei der CAP und die Häufigkeit der unnötigen Antibiotika-Verschreibung bei tiefen Atemwegsinfektionen (z. B. bei Tracheobronchitis) signifikant reduziert werden kann [10-13].

Unsere Studien zeigen, dass kardiovaskuläre Biomarker eine bessere prognostische Wertigkeit haben als inflammatorische Biomarker. Für die frühzeitige Diagnose einer relevanten kardiovaskulären Begleiterkrankung, die oftmals bei der Diagnose einer CAP nicht bekannt ist, wäre es daher sinnvoll, kardiovaskuläre Biomarker in die Diagnostik der CAP zu integrieren. Dies könnte eventuell die im Vergleich zur 28-Tages-Mortalität doppelt so hohe Langzeitmortalität (nach 6 Monaten) reduzieren.
Die pathophysiologischen Abläufe einer Infektionskrankheit sind zu komplex, um sie mit einem einzelnen Biomarker für Diagnose, Therapieentscheidung oder Prognose adäquat abbilden zu können. Durch die Kombination mehrerer Biomarker, die jeweils andere pathophysiologische Aspekte erfassen, wie zum Beispiel PCT für bakteriell induzierte Inflammation und das multiplexe proADM für Inflammation und Zirkulation, besteht in Zukunft das Potenzial, eine verbesserte Diagnostik und Differenzialdiagnose sowie Risikoabschätzung bei CAP zu erreichen.

\section{Interessenkonflikt}

$\nabla$

Die Autoren geben an, dass kein Interessenkonflikt besteht.

\section{Literatur}

1 Krüger S, Ewig S, Papassotiriou J et al. and The CAPNETZ Study group. Inflammatory parameters predict etiologic patterns but do not allow for individual prediction of etiology in patients with CAP - Results from the German competence network CAPNETZ. Respiratory Research 2009; 10: 65

2 Bauer TT, Ewig S, Marre R et al., The CAPNETZ study group. CRB-65 predicts death from community-acquired pneumonia. J Intern Med 2006; 260: $93-101$

3 Krüger S, Ewig S, Kunde J et al., CAPNETZ study group. Pro-vasopressin (copeptin) in patients with community-acquired pneumonia - influence of antibiotic pre-treatment: results from the German competence network CAPNETZ. J Antimicrob Chemother 2009; 64: 159-162

4 Krüger S, Ewig S, Kunde J et al., and The CAPNETZ Study group. Assessment of inflammatory markers in patients with community-acquired pneumonia - influence of antimicrobial pre-treatment. Results from the German competence network CAPNETZ. Clin Chim Acta 2010 Aug 7 [Epub ahead of print]

5 Krüger S, Kunde J, Hanschmann A et al. Influence of antibiotic pre-treatment on pro-atrial natriuretic peptide in community-acquired pneumonia. Eur Respir J 2008; 32 (Suppl.): 383s

6 Krüger S, Papassotiriou J, Marre R et al. Pro-atrial natriuretic peptide and pro-vasopressin to predict severity and prognosis in communityacquired pneumonia. Results from the German competence network CAPNETZ. Intensive Care Med 2007; 33: 2069-2078

7 Krüger S, Ewig S, Marre R et al. Procalcitonin predicts patients at low risk of death from community-acquired pneumonia. Eur Resp J 2008; 31: $349-355$

8 Krüger S, Ewig S, Kunde J et al. and The CAPNETZ Study group. Pro-atrial natriuretic peptide and pro-vasopressin to predict short- and longterm survival in community-acquired pneumonia. Results from the German competence network CAPNETZ. Thorax 2010; 65: 208-214

9 Krüger S, Ewig S, Giersdorf S et al. and The CAPNETZ Study group. Cardiovascular and inflammatory biomarkers to predict short- and longterm survival in community-acquired pneumonia. Results from the German competence network CAPNETZ. Am J Respir Crit Care Med 2010; Jul 16 [Epub ahead of print]

10 Burkhardt O, Ewig S, Haagen $U$ et al. A simple procalcitonin-guided strategy results in safe reductions of antibiotic use in patients with symptoms of acute respiratory tract infections in primary care. Eur Respir J 2010; Feb 25 [Epub ahead of print]

11 Christ-Crain M, Jaccard-Stolz D, Bingisser R et al. Effect of procalcitoninguided treatment on antibiotic use and outcome in lower respiratory tract infections: cluster-randomised, single-blinded intervention trial. Lancet 2004; 363: 600-607

12 Christ-Crain M, Stolz D, Bingisser R et al. Procalcitonin guidance of antibiotic therapy in community-acquired pneumonia: a randomized trial. Am J Respir Crit Care Med 2006; 174: 84-93

13 Schuetz P, Christ-Crain M, Thomann $R$ et al. Effect of procalcitoninbased guidelines vs standard guidelines on antibiotic use in lower respiratory tract infections: the ProHOSP randomized controlled trial. JAMA 2009; 302: 1059-1066 